\title{
Repeated ethanol exposure increases anxiety-like behaviour in zebrafish during withdrawal
}

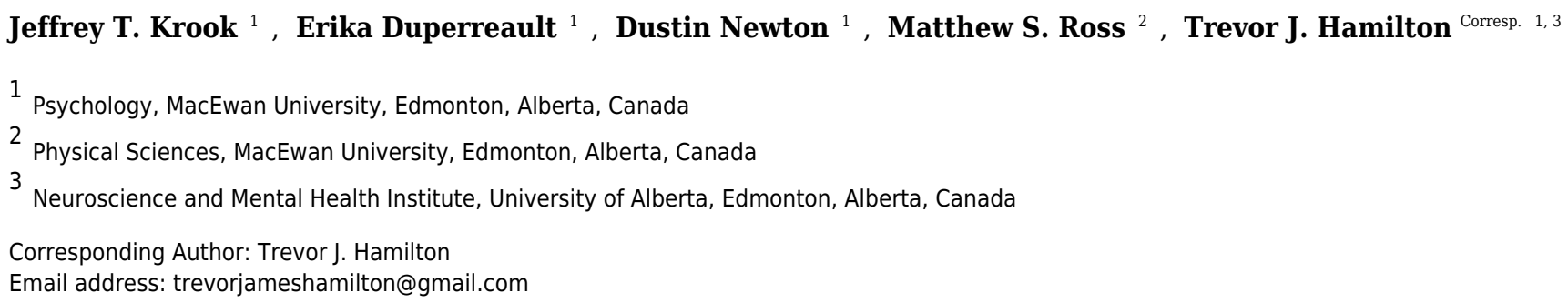

Zebrafish (Danio rerio) are quickly becoming an important model organism in behavioural neuroscience and drug addiction research. Conditioned place preference studies show that drugs of abuse produce responses in zebrafish that are similar to mammalian animal models. Repeated administration of ethanol in zebrafish results in varied withdrawalinduced behavioural responses that vary with dose and exposure duration, requiring additional investigation. Here we examine the effects of ethanol withdrawal on anxiety-like behaviours in adult zebrafish after a 21 -day ethanol dosing schedule at either $0.4 \%$ or $0.8 \%$. Anxiety-like behaviour was measured with the novel object approach test; this test involves placing a fish in a circular arena with a novel object in the center and observing the amount of exploration of the object. We found increased anxiety-like behaviour during ethanol withdrawal. This study adds to the growing body of literature that validates the zebrafish as a model organism in the field of behavioural neuroscience and addiction. 
1 Title: Repeated ethanol exposure increases anxiety-like behaviour in zebrafish during

2 withdrawal

4 Authors:

5 Jeffrey Krook ${ }^{1}$, Erika Duperreault ${ }^{1}$, Dustin Newton ${ }^{1}$, Matthew S. Ross ${ }^{2}$, Trevor J. Hamilton ${ }^{1,3 *}$, 6

7 1.Department of Psychology, MacEwan University, Edmonton, AB, Canada, T5J 4S2.

8 2.Department of Physical Sciences, MacEwan University, Edmonton, AB, Canada, T5J 4S2.

9 3. Neuroscience and Mental Health Institute, University of Alberta, Edmonton, AB, Canada, 10 T6G $2 \mathrm{H} 7$.

$11 *$ Corresponding author: trevorjameshamilton@gmail.com

\section{Abstract:}

Zebrafish (Danio rerio) are quickly becoming an important model organism in

behavioural neuroscience and drug addiction research. Conditioned place preference studies

17 show that drugs of abuse produce responses in zebrafish that are similar to mammalian animal models. Repeated administration of ethanol in zebrafish results in varied withdrawal-induced

19 behavioural responses that vary with dose and exposure duration, requiring additional

20 investigation. Here we examine the effects of ethanol withdrawal on anxiety-like behaviours in

21 adult zebrafish after a 21 -day ethanol dosing schedule at either $0.4 \%$ or $0.8 \%$. Anxiety-like

22 behaviour was measured with the novel object approach test; this test involves placing a fish in a

23 circular arena with a novel object in the center and observing the amount of exploration of the 
24 object. We found increased anxiety-like behaviour during ethanol withdrawal. This study adds

25 to the growing body of literature that validates the zebrafish as a model organism in the field of

26 behavioural neuroscience and addiction.

27

28

29

30

31

\section{Introduction:}

Alcohol (ethanol) is amongst the most commonly abused legal substances worldwide (World Health Organization, 2011). Excessive and repeated alcohol use is known to cause significant impairments in behaviour and physiology. Many of the detriments of alcohol use come from repeated exposure due to an addiction called alcohol use disorder (Levinthal \& Hamilton, 2015; American Psychiatric Association, 2013). Alcohol use disorder can be characterized by several symptoms: an impulse to seek out and consume alcohol, inhibition of controlled consumption, and the emergence of a negative emotional state during withdrawal (Vendruscolo \& Roberts, 2014), as well as many negative physical manifestations leading to increased mortality (Kendler et al., 2016). Along with the severe health and behavioural detriments, alcohol abuse is also a societal and economic burden that is undertreated (Holt \& Tobin, 2018). In part, the difficulty lies in the fact that individuals habituated to alcohol consumption that refrain from alcohol use will often experience strong aversive effects of withdrawal, such as increased stress, motivating them to resume alcohol consumption (Becker, 2012).

In order to understand withdrawal behaviours, an understanding of the mechanism through which ethanol acts on the brain is needed. Acute ethanol administration increases the activity of the inhibitory neurotransmitter $\gamma$-Aminobutyric acid (GABA) at the $\mathrm{GABA}_{\mathrm{A}}$ receptor (Faingold, N'Gouemo, \& Riaz, 1998). Ethanol agonistically activates GABA $_{\mathrm{A}}$ receptors, causing 
49 a net reduction on the excitability of neurons (Gilpin \& Koob, 2008). Due to the inhibitory nature

50 of $\mathrm{GABA}_{\mathrm{A}}$ receptors, the result of ethanol exposure in the central nervous system (CNS) is a

51 reduction in anxiety-like symptoms (Gilpin \& Koob, 2008). This ethanol-induced reduction in

52 anxiety contributes to the addictive nature of ethanol. A further contributing factor to the

53 addictive nature of ethanol is the upregulation of the dopaminergic system during ethanol

54 exposure (Chatterjee \& Gerlai, 2009). Dopamine release is associated with a pleasurable

55 response, reinforcing the motivation to abuse ethanol. Further contributions to ethanol addiction

56 come from the stimulation of the opioid system through endogenous opioids, as well as the

57 release of serotonin and glutamate (Gilpin \& Koob, 2008). However, it is neuroadaptation of the

$58 \mathrm{GABA}_{\mathrm{A}}$ receptor to the continued presence of alcohol that results in the increased anxiety,

59 delirium tremens and seizures experienced during alcohol withdrawal that make withdrawal a

60 potentially lethal occurrence (Silberman et al., 2009).

61

The zebrafish (Danio rerio) is an ideal model organism for exploring the effects of

alcoholism and withdrawal because of the ease of dosing large numbers of zebrafish in an identical manner, the sequenced genome for potential genetic manipulations (Howe et al., 2013), and the presence of analogous neurotransmitter systems to humans that mediate ethanol responses within the CNS (Gerlai et al., 2009). Studies on acute ethanol exposure in zebrafish demonstrate that ethanol has anxiolytic properties (Mathur \& Guo, 2011; Johnson \& Hamilton, 2015; Hamilton et al., 2017). Several of the reported behaviours associated with the reduction in anxiety-like behaviours due to acute (1\%) ethanol exposure in zebrafish are: increased time swimming near the top of the tank, reduced jumping, more transitions to the top of the tank, and reduced anti-predatory responses (Tran et al. 2016). However, it is important to note that with 
72 longer durations of ethanol exposure (1 hour) the anxiolytic effect on behaviour seems to change

73 to an anxiogenic state (Rosemburg et al., 2012; Mocelin et al., 2018).

74

75

76

77 78

Studies examining chronic ethanol exposure in zebrafish have reported inconsistent results, possibly due to the many different exposures times, duration of exposure, duration of withdrawal, and concentration of ethanol used (da Silva Chaves et al., 2018). To date there have been many studies examining the impact of chronic ethanol exposure (e.g. Müller et al., 2017;

Tran et al., 2015; Wong et al., 2010), and yet only a few that have examined withdrawal behaviour (Holcombe et al., 2013; Cachat et al., 2010, Mathur \& Guo, 2011; Tran et al., 2016;

Pittman \& Hylton, 2015; Gerlai et al., 2009; Pittman \& Ichikawa, 2013; Müller et al., 2017;

Dewari et al., 2016; Benneh et al., 2017). For example, a study by Müller et al. (2017) examined the effects of repeated ethanol exposure over the course of 8 days, at 20 minutes per day, and a dose of $1 \% \mathrm{v} / \mathrm{v}$. They found a significant increase in anxiety on the ninth day (one day of withdrawal from ethanol) using a test of shoaling behaviour in groups of zebrafish. In a similar study, Cachat et al. (2010) examined effects of ethanol withdrawal following 7 days of repeated ethanol dosing at a dose of $0.3 \% \mathrm{v} / \mathrm{v}$ for 20 minutes per day. Using whole body cortisol levels, which are known to correlate closely with withdrawal-evoked anxiety (Keedwell, Poon, Papadopoulos, Marshall, \& Checkley, 2001; Nava et al., 2007), the authors observed a significant elevation in cortisol levels during ethanol withdrawal (Cachat et al., 2010). Although the authors did not observe overt behavioural changes at the levels of ethanol exposure tested, it is likely there was an increase in stress and anxiety, as suggested by the increased cortisol levels (Cachat et al., 2010). This lack of behavioural change may be explained by the finding that 
94 withdrawal, although typically developing 6-24 hours after cessation of ethanol consumption,

95 may be delayed for up to 5 days (Mathur \& Guo, 2011).

96

97 Tests of anxiety-like behaviour during ethanol withdrawal in zebrafish demonstrate

98 increased anxiety in the novel tank diving test (Mathur \& Guo, 2011; Tran et al., 2015; Pittman

99 \& Hylton, 2015; Cachat et al., 2010; Pittman \& Ichikawa, 2013; Benneh et al., 2017), increased

100 anxiety in the shoaling test (Gerlai et al., 2009; Müller et al., 2017), and increased anxiety in the

101 light/dark test (Mathur \& Guo, 2009; Pittman \& Ichikawa, 2013) (for review see da Silva Chaves

102 et al., 2018). However, previous research from our lab demonstrated increased light preference in

103 a light/dark test during withdrawal after a 21-day exposure to repeated intermittent ethanol

104 exposure $(0.2 \%)$ (Holcombe et al., 2013). Usually increased light preference is associated with

105 decreased anxiety which can be caused by anxiolytic drugs (Maximino et al., 2014). However,

106 we suggested that this light preference during ethanol withdrawal was due to a conditioned-place

107 preference as the fish were dosed with ethanol in a well-lit environment with white partitions

108 around the dosing tanks that were similar to the white walls in the light/dark test, but this was not

109 empirically tested.

110 To further investigate whether repeated ethanol exposure produces increased anxiety

111 during withdrawal, in this study we exposed zebrafish to one hour of ethanol exposure $(0.4 \%$ or

$1120.8 \% \mathrm{v} / \mathrm{v}$ ) per day, for 21 days, and then tested anxiety-like behaviour after two days of

113 withdrawal. We used the novel object approach test, a validated test for anxiety in zebrafish

114 (Stewart et al., 2012, Johnson \& Hamilton 2017, Hamilton et al., 2017) and measured anxiety-

115 like behavioural responses and locomotion. Specifically, we measured: the time spent in different

116 zones of the arena, immobility, and velocity of each fish during the novel object approach test. 


\section{Materials and Methods:}

\section{Animals and Housing}

121

Adult wild-type (short-fin) zebrafish of mixed sexes ( $~ 50: 50$ males and females, $\mathrm{n}=58)$

were purchased from Aquatic Imports (Calgary, AB) and were quarantined for 60 days (same water quality parameters as below) prior to being moved to the habitat. Fish were randomly organized into groups of a maximum of 10 fish per 3L polypropylene tank in a 3-tier bench-top habitat system (Aquatic Habitats, Aquatic Ecosystems, Inc. Apopka, FL, USA). Habitat water salt, sodium bicarbonate, and acetic acid. Husbandry was as described previously (Holcombe et al., 2013, May et al., 2016). Zebrafish were maintained on a 12 hours light/12 hour dark

130 photoperiod, with lights on at 8 AM and off at 8 PM (Hamilton et al., 2016; May et al., 2016;

131 Rimstad et al., 2017; Tsang et al., 2018). Fish were fed fish pellets (Gemma Micro 300,

132 Skretting by Nutreco, France) and dry brine shrimp (Omega One Freeze Dried Mysis Shrimp

133 nutri-treat, OmegaSea Ltd., Germany) once per day, at least 30 minutes following dosing or

134 behavioural testing. All experiments were approved by the MacEwan University Animal

135 Research Ethics Board (AREB) under protocol number 05-12-13, in compliance with the

136 Canadian Council for Animal Care (CCAC) guidelines for the care and use of experimental

137 animals. All fish were experimentally naïve. 
$1410.8 \%$ ethanol (vol/vol) for 21 consecutive days between the hours of 8 A.M. and 4 P.M. The 21-

142 day exposure period was used based on a previous study conducted in this lab (Holcombe et al.,

143 2013). Zebrafish were randomly netted from the large quarantine tank and assigned to one of two

144 replicate tanks per condition with 9-10 fish per tank ( 50:50 males and females). All groups

145 were kept in separate 3L tanks in the aquatic habitat, housed on the same tier (second from the

146 top) in the middle of the row with adjacent tanks containing equal numbers of fish. Within each

147 tank a spawning insert was used to transfer fish between habitat tanks to dosing tanks (Holcombe

148 et al., 2014). Dosing tanks were prepared by adding ethanol directly to dosing tanks containing

149 habitat water, to reach a concentration of either $0.4 \%$ or $0.8 \%$ ethanol in each dosing tank

150 (Holcombe et al., 2014). After the dosing tanks were prepared the fish were transferred from the

151 habitat tanks. Dosages were based on other studies using similar ethanol exposure times and

152 concentrations (Holcombe et al., 2013; Mathur \& Guo, 2011).

153

154 Daily ethanol exposure was set to 1 hour, as internal ethanol concentrations approach a

155 steady state within 60 minutes of exposure (Tran, Chatterjee, Gerlai, 2015). This method of

156 intermittent repeated ethanol administration is preferred over a continuous ethanol exposure

157 method, which involves administering ethanol directly to the habitat tanks of the test subjects for

158 an extended period of time. The intermittent method allows greater translational relevance as it

159 more closely mirrors the repeated and intermittent consumption patterns in humans (Tran et al.

160 2016, Müller et al., 2017). After dosing, zebrafish were transferred back to their habitat tanks.

161 The control groups underwent an identical procedure, except the water they were transferred into

162 consisted of only habitat water. Throughout the duration of dosing, water was maintained at a 
163 temperature of $26-28^{\circ} \mathrm{C}$ using heating pads (Hydrofarm, USA). The dosing area was isolated

164 using white corrugated plastic barriers throughout the dosing period to minimize any external

165 visual stimuli. Behavioural testing was conducted two days after the last dose, allowing for two

166 days of withdrawal between dosing and behavioural testing. Researchers were not blind to the

167 treatment groups during dosing or testing.

168

169 Behavioural Testing

The novel object approach test was used to measure locomotion and anxiety-like

172 behaviour in individual zebrafish (Fig. 1a,b). The arena used was an opaque, white plastic

173 cylinder (34 cm diameter, $15 \mathrm{~cm}$ walls) with a water depth of $6 \mathrm{~cm}$ (Ou et al., 2015; Johnson \&

174 Hamilton, 2017). The water was maintained at $26-28^{\circ} \mathrm{C}$ using electric heating pads placed

175 underneath the arena. Behavioural testing was conducted between 8 A.M. and 4 P.M. Fish were

176 transferred from the main habitat to the experiment room by moving their $3 \mathrm{~L}$ polypropylene

177 habitat tank and placing them on heating mats in the experiment room, and allowing fish to

178 acclimate to the testing room for a minimum of 10 minutes prior to behavioural testing.

179 Following acclimation, subjects were individually netted and placed in the testing arena. Fish

180 were released into the arena facing the novel object; the object for this test was a $5 \mathrm{~cm}$ tall Lego

181 figurine (Fig.1a; Ou et al., 2015; Johnson \& Hamilton, 2017; Hamilton et al., 2017) and the

182 location of the fish was recorded for a period of 15 minutes (Hamilton et al., 2014). In order to

183 record the movement of individual fish during behavioural trials, the differencing method on

184 Ethovision XT (version 10, Noldus, VA, USA) motion tracking software was used. The

185 dependent variables measured during the test were: time spent in the center (near the object), 
186 transition, and thigmotaxis (near the wall) zones, as well as velocity $(\mathrm{cm} / \mathrm{s})$ and immobility,

187 defined as the percent change in the pixels of the fish from frame to frame, set at a threshold of

188 5\% (Pham et al., 2009). Luminance in the arenas was measured with a cal SPOT 401

189 photometer (Cooke Corp. CA, USA) and was $33 \mathrm{~cd} / \mathrm{m}^{3}$. Fish were euthanized immediately

190 following behavioural testing by immersion into an MS-222 solution.

191

192 Statistical Analysis

193 Statistical significance was determined with an alpha of $p<0.05$, and a $95 \%$ confidence

194 interval. All data sets were assessed for normality using a D’Agostino and Pearson omnibus

195 normality test. In order to examine differences between control and experimental groups,

196 parametric data was assessed using unpaired $t$-tests or one-way ANOVA with Bonferroni's

197 multiple comparison post-hoc tests. Data that were not normally distributed were examined using

198 Kruskal-Wallis tests with Dunn's multiple comparison post hoc tests. In order to compare the

199 two replicate groups from each prescribed ethanol exposure condition, we used $t$-tests and Mann-

200 Whitney tests. We found no significant differences between groups, allowing us to pool the data.

201 Behavioural data was analyzed GraphPad Prism software (Version 6, San Diego, CA, USA).

202 Data from fish with over 300 seconds of immobility were removed from the analysis (control, $203 \mathrm{n}=2 ; 0.4 \%, \mathrm{n}=4 ; 0.8 \%, \mathrm{n}=1)$. All data are presented as mean \pm S.E.M.

205 Results:

206

207 Repeated Ethanol Exposure: time in zones 

behaviour, the time spent in the center, transition, and thigmotaxis zones of the arena were quantified. Fish exposed to $0.8 \%$ ethanol spent less time in the center zone of the arena, near the

211 novel object (Fig.2a; control: $9.7 \pm 2.3 \mathrm{~s}, \mathrm{n}=18 ; 0.4 \%: 10.8 \pm 2.2 \mathrm{~s}, \mathrm{n}=16 ; 0.8 \%: 2.8 \pm 0.62 \mathrm{~s}$,

$212 \mathrm{n}=17 ; F(2,48)=5.089, \mathrm{P}=0.0099)$. We did not observe a significant difference in time spent in 213 each of the transition (Fig. 2b; control: $204.4 \pm 29.6 \mathrm{~s}, \mathrm{n}=18 ; 0.4 \%: 216.2 \pm 31.4 \mathrm{~s}, \mathrm{n}=16 ; 0.8 \%$ :

$214174.6 \pm 41.0 \mathrm{~s}, \mathrm{n}=17 ; H(3,51)=2.803, \mathrm{P}=0.2462)$, or thigmotaxis (Fig. 2c; control: $685.7 \pm$ $21530.8 \mathrm{~s}, \mathrm{n}=18 ; 0.4 \%: 672.9 \pm 32.5 \mathrm{~s}, \mathrm{n}=16 ; 0.8 \%: 722.5 \pm 40.9 \mathrm{~s}, \mathrm{n}=17 ; H(3,51)=3.217, \mathrm{P}=$ 2160.2002 ) zones following ethanol exposure, at either $0.4 \%$ or $0.8 \%$ ethanol exposure conditions 217 compared to controls.

Repeated Ethanol Exposure: locomotion immobility. There were no significant differences in velocity (Fig. 3a; control: $8.7 \pm 0.4 \mathrm{~cm} / \mathrm{s}$, $\mathrm{n}=18 ; 0.4 \%: 9.1 \pm 0.8 \mathrm{~cm} / \mathrm{s}, \mathrm{n}=16 ; 0.8 \%: 9.8 \pm 0.8 \mathrm{~cm} / \mathrm{s}, \mathrm{n}=17 ; H(3,51)=0.8916, \mathrm{P}=0.6403$ or $\mathrm{s}, \mathrm{n}=17 ; H(3,51)=2.924, \mathrm{P}=0.2317)$ between either treatment group and control fish.

\section{Discussion:}


231 ethanol exposure increases anxiety-like behaviour in zebrafish during withdrawal. Specifically,

232 during withdrawal from repeated daily doses of $0.8 \%$ ethanol, the time spent near the novel

233 object decreased significantly compared to controls, indicative of an anxiogenic response (Fig.

$2342 a)$.

235

236 To better understand the mechanisms behind the neuroadaptations caused by repeated

237 ethanol administration, the use of animal models is essential, and the zebrafish model is

238 becoming increasingly popular. To date, the analysis of anxiety-like behaviour after repeated or

239 chronic ethanol exposure has been described (Mathur \& Guo, 2011; Tran et al., 2015; Pittman \&

240 Hylton, 2015; Cachat et al., 2010; Pittman \& Ichikawa, 2013; Gerlai et al., 2009; Müller et al.,

241 2017; Holcombe et al., 2013, 2014). Notably, Mathur and Guo (2011) used 20-minute doses of

$2421 \%$ ethanol for 8 days, then used the novel tank diving test and the light/dark choice test to

243 examine anxiety-like behaviours. Interestingly, in this study the anxiety-like behaviours were not

244 observed in the first or second day of withdrawal, whereas we observed significant changes in

245 anxiety-like behaviours within two days of withdrawal. However, Mathur and Guo (2011) found

246 that after 6 days of withdrawal there was decreased bottom dwelling time in the novel tank

247 diving test and increased dark preference in the light/dark test after 7 days of withdrawal; both

248 indications of increased anxiety (Mather \& Guo, 2011). It is likely that we observed anxiogenic

249 effects earlier (at two days of withdrawal) due to the greater length of daily exposure (i.e.-60

250 minutes as opposed to 20) and/or the duration of repeated exposures (i.e.-21 days versus 8 days).

251 Another possible explanation is the difference in strain of zebrafish used; AB strain (Mathur and

252 Guo, 2011) compared to wild-type in our study and others (ex. Rosemberg, 2012; Mocelin et al.,

253 2018; Müller et al., 2017). After 8 days of exposure to 1\% ethanol for 20 minutes per day, social 
254 behaviour (inter-fish distance and farthest neighbor distance) was decreased on day 9, consistent

255 with a tighter shoal and increased anxiety. Interestingly, this experiment was performed on wild-

256 type zebrafish of mixed genders, much like the present study, and may indicate this

257 heterogeneous genetic lineage undergoes withdrawal quicker than the AB strain (Mathur and 258 Guo, 2011).

259 The candidate mechanism to explain how withdrawal from ethanol exposure is related to 260 anxiety-like behaviour is neurochemical changes involving the GABAergic system, specifically

$261 \mathrm{GABA}_{\mathrm{A}}$ receptors, resulting in increased behavioural excitability (Gilpin \& Koob, 2008). Due to

262 the constant presence of ethanol activating $\mathrm{GABA}_{\mathrm{A}}$ receptors, the mammalian nervous system

263 compensates, altering the action of GABA-induced hyperpolarization by changing the subunit

264 composition (Cagetti et al., 2003) and function (Kang et al., 1998) of GABA $A_{A}$ receptors. During

265 ethanol withdrawal, there is less inhibition of excitatory activity by $\mathrm{GABA}_{\mathrm{A}}$ receptors compared

266 to the basal state, leading to a hyperexcitable CNS (Cagetti et al., 2003). Therefore, normal levels

267 of stimulation are likely to cause over-excitation of the CNS, due to the reduced suppression of

268 the CNS, and resulting in the anxiogenesis observed during withdrawal. In the rodent chronic

269 intermittent ethanol (CIE) model, increased activity and anxiety is observed (Cagetti et al.,

270 2003), similar to increased anxiety-like behaviour in zebrafish studies (da Silva Chaves et al.,

271 2018). The contribution of the GABAergic system to the increased anxiety caused by ethanol

272 withdrawal in zebrafish is less clear. Mahabir and colleagues (2018) have recently reported no

273 changes in neurochemicals (glutamate, GABA, aspartate, glycine, taurine) up to 102 days post

274 fertilization after short embryonic ethanol exposure ( $2 \mathrm{hr}$ ), whereas reductions of dopamine,

275 DOPAC, and serotonin have been observed in the AB but not TU strain (Mahabir et al., 2014). 
276 This again highlights strain-specific differences and suggests future studies should examine

277 neurochemical changes in wild-type zebrafish after repeated ethanol exposure.

278 Another possible mechanism for the anxiogenic state induced by repeated ethanol

279 exposure in zebrafish is an increase in oxidative stress in the brain. As described above, repeated

280 ethanol exposure for 8 days (20 minutes per day), results in altered social behaviour after one

281 day of withdrawal (Müller et al., 2017). This coincides with biochemical changes in the brain,

282 including the decrease in superoxide dismutase (SOD) and catalase (CAT) enzymatic activity,

283 leading to increased reactive oxygen species and potential oxidative damage via increased lipid

284 peroxidation (Müller et al., 2017). Consistent with this is a study by Agostini and colleagues

285 (2018) that found increasing ROS (CAT/SOD ratio) at 7, 14, and 28 days of ethanol ( $0.5 \%)$

286 exposure compared to controls. In our study, exposure to the lower concentration of $0.4 \%$ caused

287 no behavioural changes, possibly due to less oxidative stress (below the threshold necessary to

288 induce behavioural changes), resulting in behaviour similar to control fish. However, the

289 previous statement is speculative as no ROS were measured, and needs experimental validation

290 in order to be confirmed in our findings. Future studies should investigate a dose-response

291 relationship between repeated ethanol administration, anxiety-like behaviour during withdrawal,

292 and resultant oxidative stress.

293 Our study further demonstrates that the novel object approach test is an appropriate test to

294 measure anxiety-like behaviour due to ethanol withdrawal. We have previously validated the use

295 of the novel object approach test to examine anxiety-like behaviour with acute $1 \%$ ethanol doses,

296 which resulted in zebrafish spending less time in the outer thigmotaxis zone and more time near

297 the novel object in the transition zone (Johnson \& Hamilton, 2017) as well as increased time in

298 the center zone near the object (Hamilton et al., 2017), indicative of decreased anxiety. Here we 
299 have shown that repeated ethanol exposure produces the opposite response during withdrawal -

300 decreased time spent near the center object - indicative of increased anxiety.

301 The novel tank diving test, light/dark preference test, predator avoidance test, and

302 shoaling test have also demonstrated increased anxiety-like behaviour following ethanol

303 withdrawal (reviewed in da Silva Chaves et al., 2018), supporting the findings reported here.

304 This contrasts our previous finding that time in the light zone was increased in the light/dark test 305 with 21-days of repeated daily ethanol exposure (0.2\%; Holcombe et al., 2013). However, in this

306 previous study (Holcombe et al., 2013) we suggest that since the fish were dosed with white

307 walls surrounding their dosing tank, we had induced a conditioned-place preference for the white

308 zone of the light/dark test, resulting in a preference for the white side of the light/dark test

309 (Holcombe et al., 2013). The current study supports this hypothesis, since we observed

310 increased anxiety-like behaviour using a different test, the novel object approach test, under the

311 similar conditions. Although this should be experimentally tested, it suggests that careful

312 consideration of the visual surroundings with repeated administration studies and the use non-

313 relevant visual cues when using the light/dark test is essential to properly assess withdrawal-

314 induced anxiety-like behaviour.

315 The present findings demonstrate an effect of repeated intermittent ethanol

316 administration-induced withdrawal and anxiogenic behavioural changes. Consistent with other

317 pharmacological studies examining zebrafish behaviour, our study supports the use of zebrafish

318 as an effective model organism for studying drug-induced withdrawal. Additionally, we have

319 found further support for the novel object approach test as an appropriate test for examining

320 anxiety-like behaviours in zebrafish. Overall, there is great translational relevance in studying the

321 anxiogenic effects of withdrawal due to repeated ethanol exposure in zebrafish. 
333

334

335

336

337

338

339

340

341

342

343

344

345

346

347

348

349

350

351 
352

353

354

355

356

357

358

359

360

361

362

363

364

365

366

367

368

369

370

371

372

373

374

375

376

377

378

379

380

381

382

383

384

385

386

387

388

389

390

391

392

393

394

395

396

\section{References:}

Agostini JF, Toé HCZD, Vieira KM, Baldin SL, Costa NLF, Cruz CU, Longo L, Machado MM, da Silveira TR, Schuck PF, Rico EP. 2018. Cholinergic System and Oxidative Stress Changes in the Brain of a Zebrafish Model Chronically Exposed to Ethanol. Neurotoxicity Research. 33: 749-758.

Becker HC. 2012. Effects of Alcohol Dependence and Withdrawal on Stress Responsiveness and Alcohol Consumption. Alcohol Research: Current Reviews 34: 448-458

Benneh CK, Biney RP, Mante PK, Tandoh A, Adongo DW, Woode E. 2017. Maerua angolensis stem bark extract reverses anxiety and related behaviours in zebrafish-Involvement of GABAergic and 5-HT systems. Journal of Ethnopharmacology. 207:129 - 145.

Cachat J, Canavello P, Elegante M, Bartels, B, Hart P, Bergner C. 2010. Modeling withdrawal syndrome in zebrafish. Behavioural Brain Research 208(2):371-376

Cagetti E, Liang J, Spigelman I, Olsen RW. 2003. Withdrawal from chronic intermittent ethanol treatment changes subunit composition, reduces synaptic function, and decreases behavioral responses to positive allosteric modulators of GABAA receptors. Molecular Pharmacology $63: 53-64$

Chatterjee D, Gerlai R. 2009. High precision liquid chromatography analysis of dopaminergic and serotoninergic responses to acute alcohol exposure in zebrafish. Behavioural Brain Research 200(1):208-213

Da Silva Chaves S, Felicio G, Costa B, de Oliveira W, Lima-maximino C. 2018. Behavioural and biochemical effects of ethanol withdrawal in zebrafish. Pharmacology Biochemistry and Behaviour 169:48 - 58

Dewari PS, Ajani F, Kushawah G, Kumar DS, Mishra RK. 2016. Reversible loss of reproductive fitness in zebrafish on chronic alcohol exposure. Alcohol 50:83-89

Faingold C, N'Gouemo, P, Riaz, A. 1998. Ethanol and neurotransmitter interactions - from molecular to integrative effects. Progress in Neurobiology 55(5):509-535

Gerlai R, Chatterjee D, Pereira T, Sawashima T, Krishnannair R. 2009. Acute and chronic alcohol dose: population differences in behaviour and neurochemistry of zebrafish. Genes, Brain and Behaviour 8:586-599

Gilpin NW, Koob GF. 2008. Neurobiology of Alcohol Dependence: Focus on Motivational Mechanisms. Alcohol Research \& Health 31(3):185-195

Hamilton TJ, Myggland A, Duperreault E, May Z, Gallup J Powell RA, Schalomon M, Digweed SM. 2016. Episodic-like memory in zebrafish. Animal Cognition 19(6): 1071 - 1079. 
397 Hamilton TJ, Holcombe A, Tresguerres M. 2014. CO2-induced ocean acidification increases 398 anxiety in rockfish via alteration of GABAA receptor functioning. Proceedings. Biological Sciences Royal Society (Great Britain) 281(1775): 20132509

Holt SR, Tobin DG. 2018. Pharmacotherapy for Alcohol Use Disorder. Medical Clinics of North America 102:653-666

Holcombe A, Howorko A, Powell RA, Schalomon M, Hamilton TJ. 2013. Reversed Scototaxis during Withdrawal after Daily-Moderate, but Not Weekly-Binge, Administration of Ethanol in Zebrafish. PLoS ONE 8(5): e63319

Holcombe A, Schalomon M, Hamilton TJ. 2014. A novel method of drug administration to multiple zebrafish (Danio rerio) and the quantification of withdrawal. Journal of Visualized Experiments 93

Howe, K., Clark, M. D., Torroja, C. F., Torrance, J., Berthelot, C., Muffato, M., Collins, J. E., Humphray, S., McLaren, K., Matthews, L., McLaren, S., Sealy, I., Caccamo, M., Churcher, C., Scott, C., Barrett, J. C., Koch, R., Rauch, G. J., White, S., Chow, W., Kilian, B., Quintais, L. T., Guerra-Assunção, J. A., Zhou, Y., Gu, Y., Yen, J., Vogel, J. H., Eyre, T., Redmond, S., Banerjee, R., Chi, J., Fu, B., Langley, E., Maguire, S. F., Laird, G. K., Lloyd, D., Kenyon, E., Donaldson, S., Sehra, H., Almeida-King, J., Loveland, J., Trevanion, S., Jones, M., Quail, M., Willey, D., Hunt, A., Burton, J., Sims, S., McLay, K., Plumb, B., Davis, J., Clee, C., Oliver, K., Clark, R., Riddle, C., Elliot, D., Threadgold, G., Harden, G., Ware, D., Begum, S., Mortimore, B., Kerry, G., Heath, P., Phillimore, B., Tracey, A., Corby, N., Dunn, M., Johnson, C., Wood, J., Clark, S., Pelan, S., Griffiths, G., Smith, M., Glithero, R., Howden, P., Barker, N., Lloyd, C., Stevens, C., Harley, J., Holt, K., Panagiotidis, G., Lovell, J., Beasley, H., Henderson, C., Gordon, D., Auger, K., Wright, D., Collins, J., Raisen, C., Dyer, L., Leung, K., Robertson, L., Ambridge, K., Leongamornlert, D., McGuire, S., Gilderthorp, R., Griffiths, C., Manthravadi, D., Nichol, S., Barker, G., Whitehead, S., Kay, M., Brown, J., Murnane, C., Gray, E., Humphries, M., Sycamore, N., Barker, D., Saunders, D., Wallis, J., Babbage, A., Hammond, S., MashreghiMohammadi, M., Barr, L., Martin, S., Wray, P., Ellington, A., Matthews, N., Ellwood, M., Woodmansey, R., Clark, G., Cooper, J., Tromans, A., Grafham, D., Skuce, C., Pandian, R., Andrews, R., Harrison, E., Kimberley, A., Garnett, J., Fosker, N., Hall, R., Garner, P., Kelly, D., Bird, C., Palmer, S., Gehring, I., Berger, A., Dooley, C. M., Ersan-Ürün, Z., Eser, C., Geiger, H., Geisler, M., Karotki, L., Kirn, A., Konantz, J., Konantz, M., Oberländer, M., Rudolph-Geiger, S., Teucke, M., Lanz, C., Raddatz, G., Osoegawa, K., Zhu, B., Rapp, A., Widaa, S., Langford, C., Yang, F., Schuster, S. C., Carter, N. P., Harrow, J., Ning, Z., Herrero, J., Searle, S. M., Enright, A., Geisler, R., Plasterk, R. H., Lee, C., Westerfield, M., de Jong, P. J., Zon, L. I., Postlethwait, J. H., Nüsslein-Volhard, C., Hubbard, T. J., Roest Crollius, H., Rogers, J., Stemple, D. L. (2013). The zebrafish reference genome sequence and its relationship to the human genome. Nature, 496(7446), 498-503.

Johnson A, Hamilton TJ. 2017. Modafinil decreases anxiety-like behaviour in zebrafish. PeerJ 5: e2994 


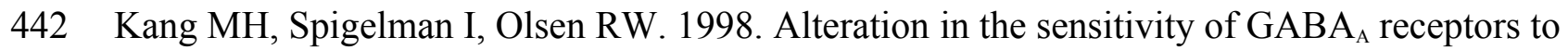
443 allosteric modulatory drugs in rat hippocampus after chronic intermittent ethanol treatment.

444 Alcoholism: Clinical and Experimental Research 22:2165-2173

445

446

447

448

449

450

451

452

453

454

455

456

457

458

459

460

461

462

463

464

465

466

467

468

469

470

471

472

473

474

475

476

477

478

479

480

481

482

483

484

485

486

Keedwell PA, Poon L, Papadopoulos AS, Marshall EJ, Checkley SA. 2001. Salivary cortisol measurements during a medically assisted alcohol withdrawal. Addiction Biology 6:247-56

Kendler, K., Ohlsson, H., Sundquist, J., Sundquist K. 2016. Alcohol use disorder and mortality across the lifespan: a longitudinal cohort and co-relative analysis. JAMA psychiatry 73(6): 575 581

Leggio L, Kenna GA, Swift RM. 2008. New developments for the pharmacological treatment of alcohol withdrawal syndrome. A Focus on non-benzodiazepine GABAergic medications.

Progress in Neuropsychopharmacology \& Biological Psychiatry 32(5): 1106 - 1117

Levinthal CF \& Hamilton TJ. 2015. Drugs, Behaviour \& Modern Society, First Canadian Edition. Pearson Education Canada.

Mahabir S, Chatterjee D, Gerlai R. 2018. Short exposure to low concentrations of alcohol during embryonic development has only subtle and strain-dependent effects on the levels of five amino acid neurotransmitters in zebrafish, Neurotoxicology and Teratology 68:91-96

Mahabir S, Chatterjee D, Gerlai R. 2014. Strain dependent neurochemical changes induced by embryonic alcohol exposure in zebrafish. Neurotoxicology and Teratology 41:1-7

Mathur P, Guo P. 2011. Differences of acute versus chronic ethanol exposure on anxiety like behavioural responses in zebrafish, Behavioural Brain Research 219:234-239

May Z, Morrill A, Holcombe A, Johnston T, Gallup J, Fouad K, Schalomon M, Hamiltom TJ. 2016. Object recognition memory in zebrafish. Behaviour Brain Research 296: $199-210$

Maximino C, da Silva AWB, Araújo J, Lima MG, Miranda V, Puty B, Benzecry R, PicancoDiniz DLW, Gouveia Jr A, Oliveira KRM, Herculano, AM. 2014. Fingerprinting of Psychoactive Drugs in Zebrafish Anxiety-Like Behaviors. PLoS ONE 9(7) e103943

Mocelin R, Marcon M, D'ambros S, Herrmann AP, da Rosa Araujo AS, Piato A 2018. Behavioral and Biochemical Effects of N-Acetylcysteine in Zebrafish Acutely Exposed to Ethanol. Neurochemical research 43:458-464. DOI: 10.1007/s11064-017-2442-2.

Müller TE, Nunes SZ, Silveira A, Loro, VL, Rosemberg DB. 2017.

Repeated ethanol exposure alters social behavior and oxidative stress parameters of zebrafish. Progress in Neuro-Psychopharmacology and Biological Psychiatry 79:105-111

Nava F, Premi S, Manzato E, Campagnola W, Lucchini A, Gessa GL. 2007. Gammahydroxybutyrate reduces both withdrawal syndrome and hypercortisolism in severe abstinent 
487 alcoholics: an open study vs. diazepam. American Journal of Drug and Alcohol Abuse 33:379$488 \quad 392$

489

490

491

492

493

494

495

496

497

498

499

500

501

502

503

504

505

506

507

508

509

510

511

512

513

514

515

516

517

518

519

520

521

522

523

524

525

526

527

528

529

530

531

532
Olsen RW, Liang J. 2017. Role of GABA receptors in alcohol use disorders suggested by chronic intermittent ethanol (CIE) rodent model. Molecular Brain 10:45

Ou M, Hamilton TJ, Eom J, Lyall EM, Gallup J, Jiang A, Lee J, Close DA, Yun S-S, Brauner CJ. 2015. Responses of pink salmon to $\mathrm{CO}_{2}$-induced aquatic acidification. Nature Climate Change 5:950-955

Pham J, Cabrera SM, Sanchis-Segura C, Wood MA. 2009. Automated scoring of fear-related behavior using EthoVision software. Journal of Neuroscience Methods 178(2):323-326

Pittman J, Hylton A. 2015. Behavioral, endocrine, and neuronal alterations in zebrafish (Danio rerio) following sub-chronic co-administration of fluoxetine and ketamine. Pharmacology Biochemistry and Behavior 139:158-162

Pittman JT, Ichikawa KM. 2013. iPhone ${ }^{\circledR}$ applications as versatile video tracking tools to analyze behavior in zebrafish (Danio rerio). Pharmacology Biochemistry and Behavior 106:137-142

Rimstad LA, Holcombe A, Pope A, Hamilton TJ, Schalomon MP. 2017. Preferences for achromatic horizontal, vertical, and square patterns in zebrafish (Danio rerio) PeerJ 5:e3748

Rosemberg DB, Braga MM, Rico EP, Loss CM, Córdova SD, Mussulini BHM, Blaser RE, Leite CE, Campos MM, Dias RD, Calcagnotto ME, de Oliveira DL, Souza DO 2012. Behavioral effects of taurine pretreatment in zebrafish acutely exposed to ethanol. Neuropharmacology 63:613-623. DOI: 10.1016/j.neuropharm.2012.05.009.

Tran S, Nowicki M, Chatterjee D, Gerlai R. 2016. Acute and chronic ethanol exposure differentially alters alcohol dehydrogenase and aldehyde dehydrogenase activity in the zebrafish liver. Progress in Neuro-Psychopharmacology and Biological Psychiatry 56:221-226 Tran S, Chatterjee D, Gerlai R. 2015. An integrative analysis of ethanol tolerance and withdrawal in zebrafish (Danio rerio). Behavioural Brain Research 276:161 - 170

Tsang B, Ansari R, Azhar A, Gerlai R. 2018. Drinking in the morning versus evening: timedependent differential effects of acute alcohol administration on the behaviour of zebrafish. Pharmacology Biochemistry and Behaviour 175: 174 - 185

Silberman Y, Bajo M, Chappell AM, Christian DT, Cruz M, Diaz MR, Kash, T, Lack AK, Messing RO, Siggins GR, Winder D, Roberto M, McCool BA, Weiner JL. 2009.

Neurobiological Mechanisms Contributing to Alcohol-Stress-Anxiety Interactions. Alcohol 43(7): 509-519

Stewart A, Gaikwad S, Kyzar E, Green J, Roth A, Kalueff AV. 2012. Modeling anxiety using adult zebrafish: a conceptual review. Neuropharmacology 62(1):135-143 
533

534 Vendruscolo LF, Roberts AJ. 2014. Operant alcohol self-administration in dependent rats: focus 535 on the vapor model. Alcohol 48: 277-286

536

537 Wong K, Elegante M, Bartels B, Elkhayat S, Tien D, Roy S, Goodspeed J, Suciu C, Tan J, 538 Grimes C Chung A, Rosenberg M. Gaikwad S, Denmark A, Jackson A, Kadri F, Chung KM, 539 Stewart A, Gilder T, Beeson E, Zapolsky I, Wu N, Cachat J, Kalueff AV. 2010. Analyzing

540 habituation responses to novelty in zebrafish (Danio rerio). Behaviour Brain Research 208:450$541 \quad 457$

542

543 World Health Organization. (2011). Global status report on alcohol and health 2011.

545 


\section{Figure Legends.}

547

548 Figure 1. Novel object approach test. (a) The novel object used in the test was a 549 multicolour LEGO figurine (height: $5 \mathrm{~cm}$, width: $1.5 \mathrm{~cm}$ ). (b) The novel object was

550 placed in the center of the circular arena (diameter: $34 \mathrm{~cm}$, wall height: $16 \mathrm{~cm}$ ). 551

552

553

554

555

556

557

558

559

560
Figure 2. Repeated ethanol administration effects on time in zones during withdrawal. Each data point represents and individual fish. (a) Time in center zone is decreased with $0.8 \%$ ethanol. (b) No effect on time spent in transition zone. (c) No effect on time spent in thigmotaxis zone. ${ }^{*} \mathrm{P}<0.05$. Mean \pm S.E.M.

Figure 3. Repeated ethanol administration effects on locomotion during withdrawal. Each data point represents and individual fish. (a) No effect on velocity. (b) No effect on immobility. Mean \pm S.E.M. 
Figure 1 (on next page)

Novel object approach test

(a) The novel object used in the test was a multicolour LEGO figurine (height: $5 \mathrm{~cm}$, width:

$1.5 \mathrm{~cm}$ ). (b) The novel object was placed in the center of the circular arena (diameter: $34 \mathrm{~cm}$, wall height: $16 \mathrm{~cm}$ ). 
a)

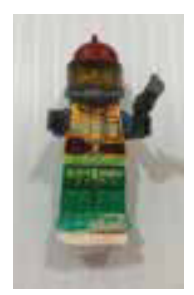

b)

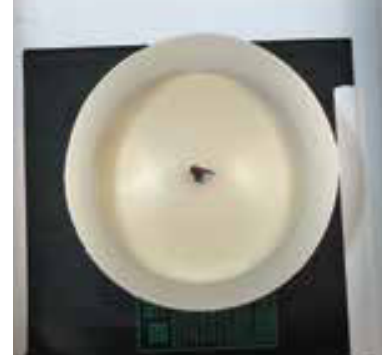




\section{Figure 2 (on next page)}

Repeated ethanol administration effects on time in zones during withdrawal.

Each data point represents and individual fish. (a) Time in center zone is decreased with $0.8 \%$ ethanol. (b) No effect on time spent in transition zone. (c) No effect on time spent in thigmotaxis zone. $* P<0.05$. Mean \pm S.E.M. 
a)

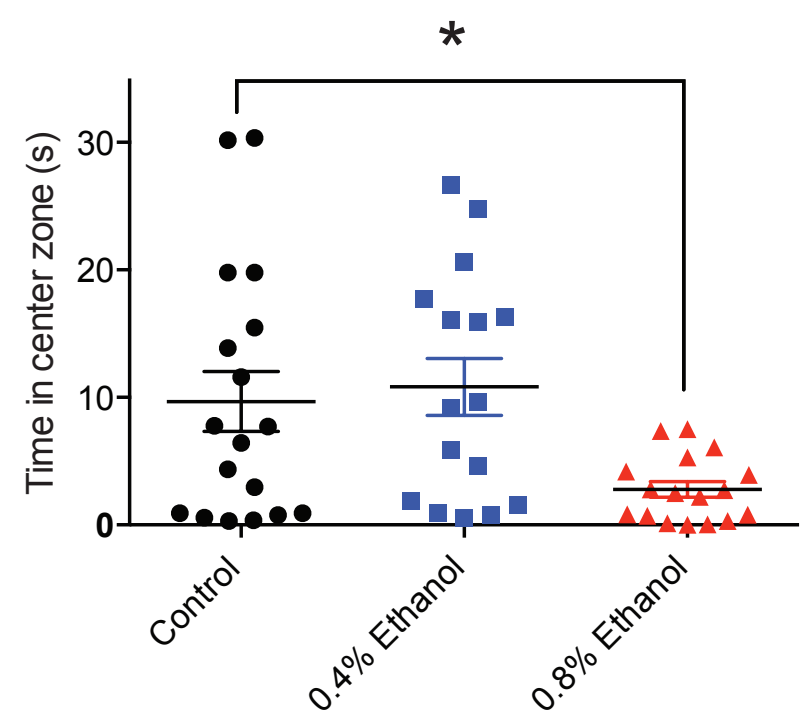

b)

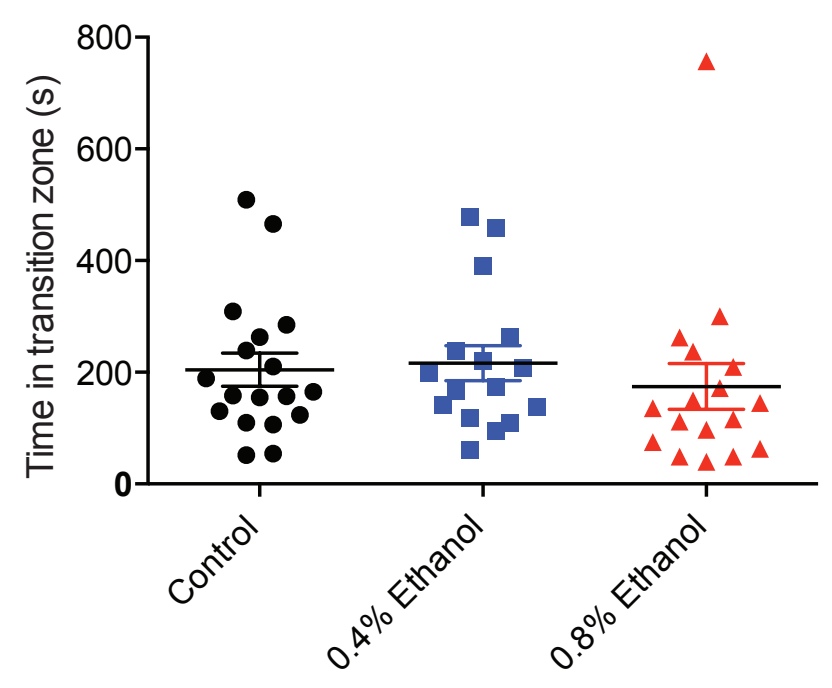

c)

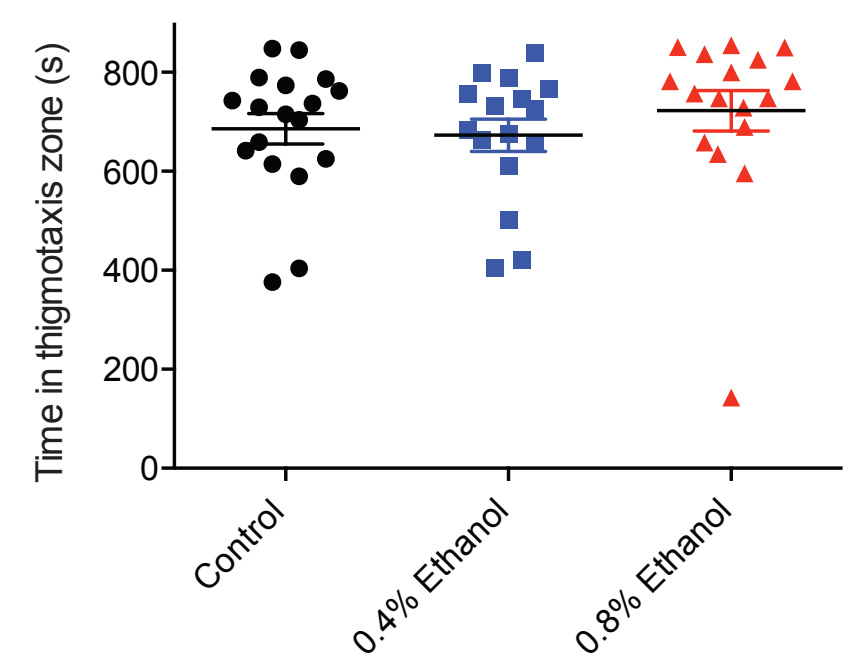




\section{Figure 3 (on next page)}

Repeated ethanol administration effects on locomotion during withdrawal.

Each data point represents and individual fish. (a) No effect on velocity. (b) No effect on immobility. Mean \pm S.E.M. 


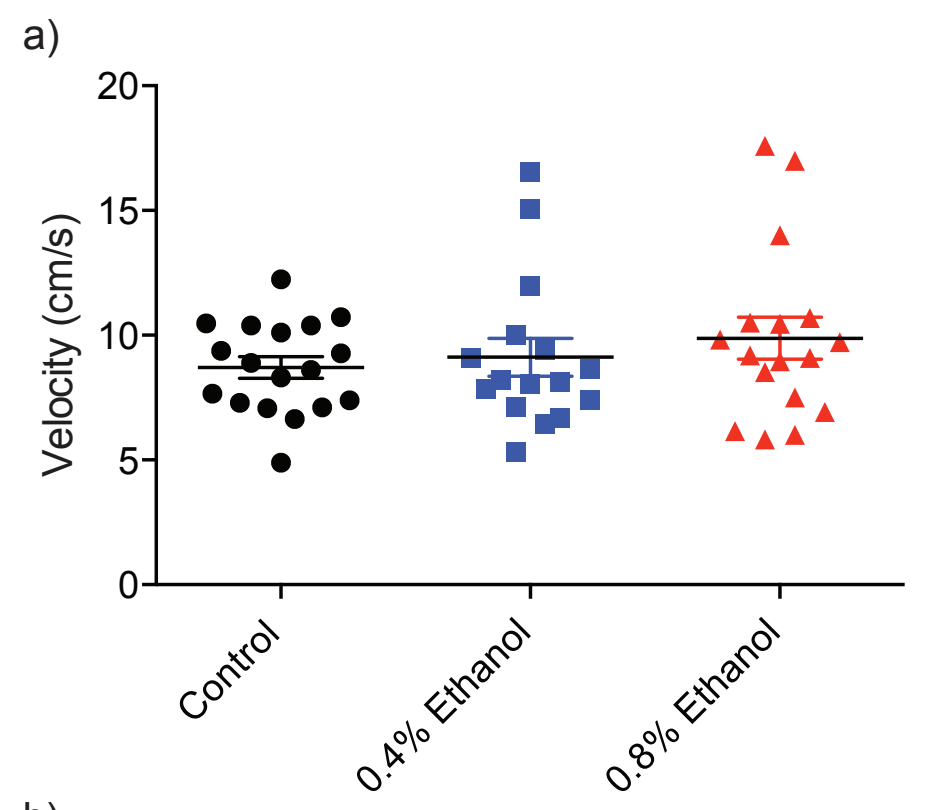

b)

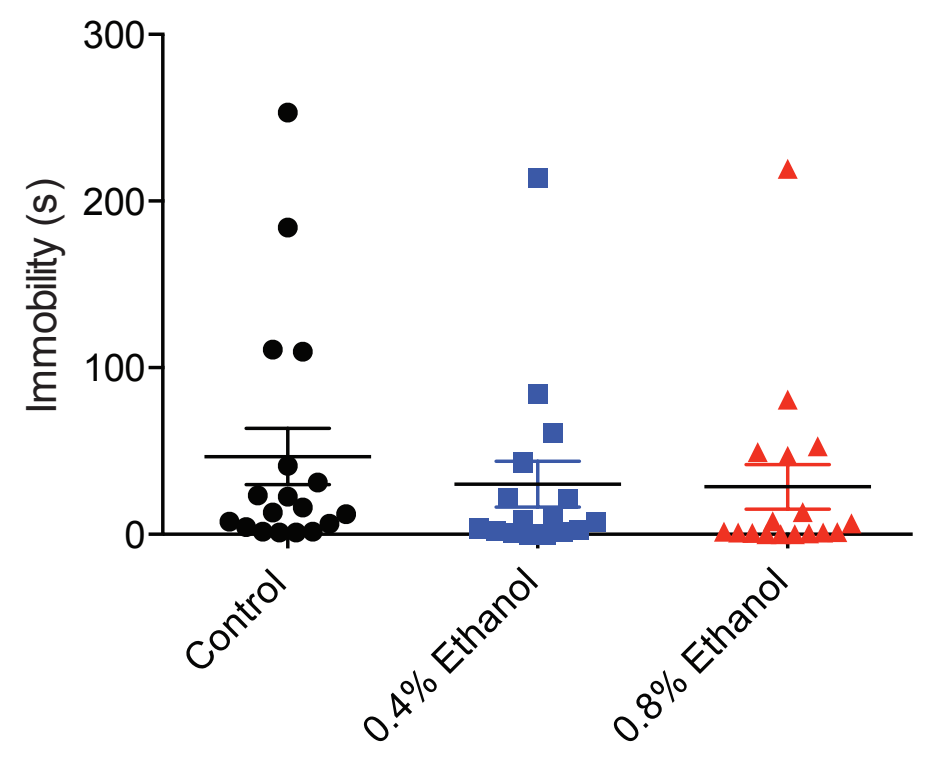

